\title{
Inquérito epidemiológico em saúde bucal e fatores psicossociais em idosos - um estudo piloto
}

\author{
Epidemiological survey on oral health and psychosocial factors \\ in the elderly - a pilot study
}

\author{
Rafael Menezes-Silva', Dhelfeson Willya Douglas de Oliveira² $₫$, Ana Clara Sá-Pinto ${ }^{3}$, Paulo Cesar Brambilla Biscaro ${ }^{4}$, \\ Natália Pinheiro Orti ${ }^{5}$, Maria Letícia Ramos-Jorge ${ }^{3}$ \\ 1 Faculdade de Odontologia de Bauru (FOB) da Universidade de São Paulo (USP). Bauru, SP, Brasil. \\ 2 Faculdade de Odontologia da Universidade Federal de Minas Gerais (UFMG). Belo Horizonte, MG, Brasil. \\ ${ }^{3}$ Departamento de Odontologia da Universidade Federal dos Vales do Jequitinhonha e Mucuri (UFVIM). Diamantina, MG, Brasil. \\ ${ }^{4}$ Centro de Cardiologia Não Invasiva de Bauru (CentroCard). Bauru, SP, Brasil. \\ ${ }^{5}$ Instituto de Análise do Comportamento (IACB). Bauru, SP, Brasil.
}

\section{RESUMO}

Objetivos: Realizar um levantamento epidemiológico da saúde bucal e fatores psicossociais associados abrangendo indivíduos com idade igual ou superior a 60 anos.

Métodos: Trata-se de um estudo transversal, com a população-alvo constituída de idosos. Foi selecionada uma amostra representativa do município de Diamantina, estado de Minas Gerais, composta por pessoas com mais de 60 anos, cadastradas nos postos da Estratégia Saúde da Família da zona rural e urbana do município. Os dados foram coletados em domicílio, por meio de exames bucais realizados por um único examinador, treinado e calibrado, e de questionários. Foi investigado o índice CPOD (média de dentes cariados, perdidos e obturados em um grupo de indivíduos), assim como dados sociodemográficos. Também foram obtidos dados sobre qualidade de vida relacionada à saúde bucal, locus de controle de saúde, consumo de álcool e drogas na senescência. Os dados obtidos foram submetidos à análise descritiva e ao teste de correlação de Spearman. O nível de significância adotado foi de $\leq 5 \%$.

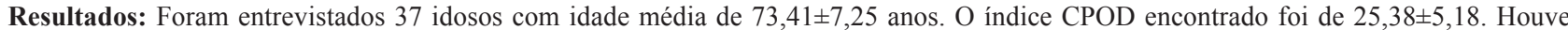
correlação estatisticamente significante entre maior pontuação no domínio limitação funcional e maior idade, maior locus externo e maior locus ao acaso, aumento na limitação funcional e diminuição da renda, maior pontuação no domínio limitação funcional e maior locus interno, maior índice CPOD e menor pontuação no domínio incapacidade social.

Conclusões: Pelo presente estudo concluiu-se que a qualidade de vida, locus de controle de saúde e índice CPOD são fatores diretamente correlacionados em idosos. Enfatiza-se a importância da atuação de profissionais de saúde e, mais especificamente, de saúde bucal, na promoção de um envelhecimento saudável.

DESCRITORES: qualidade de vida; saúde bucal; locus de controle de saúde; índice CPOD; idoso.

\section{ABSTRACT}

Aims: To conduct an epidemiological survey on oral health and psychosocial factors in individuals aged 60 years or older.

Methods: This is a cross-sectional study with elderly individuals as the target population. A representative sample of Diamantina, a town in Minas Gerais State, southeastern Brazil, was used. The sample consisted of individuals aged over 60 years registered at Family Health Strategy units in the rural and urban areas of Diamantina. Data were collected at home by means of oral examinations performed by a single trained and calibrated examiner, and through questionnaires. The decayed, missing and filled teeth (DMFT) index and sociodemographic data were investigated. Data on oral health-related quality of life, health locus of control, and use of alcohol and drugs in old age were also obtained.

Results: Thirty-seven elderly individuals with a mean age of $73.41 \pm 7.25$ years were interviewed. The average DMFT index was equal to $25.38 \pm 5.18$. There was a statistically significant correlation between functional limitations and age, external locus and random locus, functional limitation and income, functional limitation and internal locus, and DMFT index and social disability.

Conclusions: It can be concluded that quality of life, health locus of control, and DMFT index are correlated factors in the elderly. Health professionals should act in seeking to promote healthy aging.

KEY WORDS: quality of life; oral health; locus of health control; DMF index; aged. 
Abreviaturas: CPOD, índice de dentes cariados, perdidos e obturados em um grupo de indivíduos; UFVJM, Universidade Federal dos Vales do Jequitinhonha e Mucuri; OHIP-14, Oral Health Impact of Profile curto; AUDIT, teste para identificação de problemas relacionados ao uso do álcool; ASSIST, teste para triagem do envolvimento com álcool, cigarro e outras substâncias; MHLC, Multidimensional Health Locus of Control Scale.

\section{INTRODUÇÃO}

A pessoa idosa é definida como o indivíduo acima de 65 anos ou mais nos países desenvolvidos e 60 anos ou mais de idade para indivíduos de países subdesenvolvidos [1]. Nas últimas três décadas, os brasileiros envelheceram em um ritmo mais acelerado devido à redução da taxa de mortalidade, ao aumento da expectativa de vida e, principalmente, à rapidez com que declinaram as taxas de fecundidade $[2,3]$. Com isso, torna-se imprescindível garantir aos idosos uma qualidade de vida, satisfação pessoal e existencial durante sua senescência, geradas através da síntese dos elementos considerados como padrão de conforto e bem-estar físico, mental e social que, consequentemente, refere-se à saúde do indivíduo [4].

Qualidade de vida é um termo multidimensional [5] e deve ser estudada de acordo com uma série de fatores que podem influenciar na sua percepção [6], como questões sociodemográficas e econômicas, condição de saúde bucal e geral, além de comportamentos de risco, como o consumo de álcool, drogas ou substâncias psicoativas. A multidimensionalidade do termo qualidade de vida é determinante para a avaliação do bem-estar e da satisfação com a saúde que está associada cada vez mais à área da saúde pública [5].

O envelhecimento populacional é um dos maiores desafios da saúde pública contemporânea, devido à demanda crescente por serviços de saúde por parte dessa população. Em geral, as doenças dos idosos são crônicas e múltiplas, perduram por vários anos e exigem acompanhamento e cuidados permanentes, medicação contínua e exames periódicos [7]. O governo deve ser atuante, de modo a promover e auxiliar o suporte familiar e, além disso, garantir o acesso pleno do idoso ao Sistema Único de Saúde de forma eficaz e efetiva.

Em relação ao consumo de álcool e drogas ilícitas, embora diminuam com a idade, as taxas podem continuar altas [8]. Isso é especialmente importante pelo fato de a população idosa ser considerada de risco. Grande atenção também deve ser direcionada ao consumo de cigarro e outras substâncias psicoativas, que são raros nessa população, mas requerem cuidados por ser esse um grupo especial [8].
Cada vez mais se procura avaliar as pessoas quanto aos comportamentos de saúde (tabagismo, consumo de bebidas alcoólicas, consumo de frutas e hortaliças, consumo habitual de carnes com excesso de gordura, adição de sal à refeição pronta e atividade física entre ouros) e seu controle (locus de controle) [9]. Crenças sobre percepção de controle de saúde são consideradas um importante fator motivacional para a compreensão da adoção de comportamentos de promoção da saúde pelos idosos [10]. O locus de controle de saúde é capaz de indicar se os indivíduos vão ou não tomar responsabilidade por sua própria saúde [11,12].

É importante que os profissionais da saúde e os responsáveis pela elaboração de políticas públicas entendam cada vez mais a complexidade do envelhecimento num país como o Brasil e tenham abertura suficiente para a construção de novos modelos que possam garantir qualidade de vida à pessoa idosa [13], utilizando todo seu conhecimento para dar resposta às suas necessidades, visando estabelecer um vínculo de respeito, solidariedade e responsabilidade. Nesse sentido, o objetivo do presente estudo foi realizar um levantamento epidemiológico da saúde bucal e fatores psicossociais associados abrangendo indivíduos com idade igual ou superior a 60 anos.

\section{MÉTODOS}

Trata-se de um estudo quantitativo observacional de corte transversal, com a população-alvo sendo constituída de idosos, de ambos os sexos e sem distinção de cor ou condição socioeconômica. O estudo piloto foi realizado em Diamantina, cidade localizada no vale do Jequitinhonha, em Minas Gerais, com população de 47.647 habitantes. O município foi dividido em nove estratos, de acordo com a cobertura das unidades básicas de saúde. Em cada estrato foi obtida a lista de todos os idosos cadastrados; os idosos que seriam investigados foram selecionados por sorteio. Assim, foi selecionada uma amostra representativa do município de Diamantina, composta por idosos acima de 60 anos, cadastrados na Estratégia Saúde da Família na zona rural e urbana de Diamantina/MG.

Foram excluídos idosos com alterações comportamentais que dificultem a coleta dos dados; pessoas hospitalizadas ou institucionalizadas e com doenças sistêmicas graves (alienação mental, cardiopatia grave, cegueira, doença de Parkinson, espondiloartrose anquilosante, estado avançado da doença de Paget, esclerose múltipla, hanseníase, nefropatia grave, neoplasia maligna, paralisia irreversível e incapacitante, 
aids, tuberculose ativa, hepatopatia grave ou fibrose cística).

O estudo foi aprovado pelo Comitê de Ética em Pesquisa com Seres Humanos da Universidade Federal dos Vales do Jequitinhonha e Mucuri (UFVJM) sob o parecer número 118/12 e foi conduzido de acordo com a Declaração de Helsinki, 1975, revisada em 2008, e com a Resolução 466/2012 do Conselho Nacional de Saúde do Brasil, publicada em 13/06/13. Todos os participantes assinaram um termo de consentimento livre e esclarecido previamente à coleta de dados.

Os dados foram coletados em domicílio, de janeiro a março de 2015. Foram realizados exames bucais e aplicados questionários de heteroavaliação. A equipe de trabalho de campo era formada por um examinador e um auxiliar responsável por anotar os dados do exame bucal e por aplicar o questionário. Os dados de todos os participantes foram coletados por esses mesmos pesquisadores. Foi realizado um treinamento prévio com o entrevistador para que o mesmo realizasse a leitura dos itens de forma clara e objetiva, sem influenciar a resposta do idoso.

O exame intrabucal foi realizado na própria residência, com luz natural e sem nenhum procedimento invasivo [14], utilizando apenas gaze, espátula de madeira e espelho. Todo esse material foi adquirido, esterilizado e transportado pelos pesquisadores responsáveis até o local do exame, conforme princípios de biossegurança vigentes e sem ônus ao participante.

Foi realizada uma calibração teórica e prática para o CPOD (índice de dentes cariados, perdidos e obturados) [15]. Um pesquisador (RMS) foi calibrado na clínica de Periodontia e Cirurgia Bucal do Departamento de Odontologia da UFVJM para a realização dos exames bucais. O valor do teste Kappa intra-examinador foi 0,95 . Para obtenção do índice CPOD, foram seguidos os critérios da Organização Mundial de Saúde [15], notadamente quanto à presença ou não de cáries em regiões de fóssulas e fissuras.

Foram coletados dados sociodemográficos e a autoavaliação de saúde. Os participantes foram investigados quanto ao sexo, idade, religião, cor, tipo de estrutura familiar, habitação, presença de saneamento básico, número de moradores da casa, tipo de escola, escolaridade, estado civil e escolaridade dos pais, renda mensal familiar, última visita ao dentista, onde procurou atendimento, se foi atendido pelo Sistema Único de Saúde, qual o motivo da procura por atendimento odontológico e avaliação do atendimento odontológico recebido. As condições de saúde bucal investigadas foram: edentulismo, oclusão dentária, presença de cárie dentária e lesões. Além disso, em relação à saúde geral, investigou- se fatores psicossociais: qualidade de vida, percepção da própria saúde, problemas relacionados ao uso de álcool e substâncias psicoativas. O tempo médio para aplicação dos questionários e exame bucal foi de duas horas para cada participante.

A avaliação de qualidade de vida relacionada à saúde bucal foi verificada através da versão brasileira validada do Oral Health Impact of Profile curto (OHIP-14), o qual apresenta 14 questões respondidas em escala tipo Likert e abrange sete dimensões: limitação funcional, dor física, desconforto psicológico, incapacidade física, incapacidade psicológica, incapacidade social e desvantagem [16]. As 14 respostas são somadas a fim de produzir uma pontuação total que pode variar de $0 \mathrm{a}$ 56 , com maiores pontuações indicando mais influência na qualidade de vida.

O teste para identificação de problemas relacionados ao uso do álcool (AUDIT) foi desenvolvido para avaliar o risco ou o abuso do consumo de álcool e principalmente para auxiliar as práticas terapêuticas [17]. A pontuação varia de 0 a 40 pontos, sendo que abaixo de 7 pontos considera- se que o indivíduo possui baixo risco a dependência do álcool, de 8 a 15 pontos um risco incerto, de 16 a 19 pontos identificamse usuários de álcool de forma perigosa e nociva, e com pontuação acima de 20 pontos classificam-se os indivíduos dependentes do álcool [18].

Foi utilizado o teste para triagem do envolvimento com álcool, cigarro e outras substâncias (ASSIST). Trata-se de um questionário contendo oito questões sobre o uso de nove classes de substâncias psicoativas (tabaco, álcool, maconha, cocaína, estimulantes, sedativos, inalantes, alucinógenos e opiáceos). As questões abordam a frequência de uso na vida e nos últimos três meses, problemas relacionados ao uso, preocupação a respeito do uso por parte de pessoas próximas ao usuário, prejuízo na realização de tarefas esperadas, tentativas mal sucedidas de eliminar ou reduzir o uso, sentimento de compulsão e uso por via injetável. Com exceção da primeira questão, que não gera nenhum valor no escore, cada resposta corresponde a um resultado, que varia de 0 a 4, sendo que a soma total pode variar de 0 a 20 . Considera-se a faixa de 0 a 3 como indicativa de uso ocasional, de 4 a 15 como indicativa de abuso e maior ou igual a 16 como sugestiva de dependência [19].

Para a determinação do Locus de Controle de Saúde, foi utilizada a escala Multidimensional Health Locus of Control Scale (MHLC) [20,21]. Nessa escala, os escores fornecem o grau em que o sujeito acredita em si mesmo, em pessoas poderosas (médico, 
dentista, mãe), e ao acaso como fonte de controle da saúde. São apresentados aos sujeitos 18 itens como uma escala única e respondidos na forma Likert. Para obter os escores, somam-se os números dos itens correspondentes. Sub-escala I: somar os itens 1, 6, 8, 12, 13, 17. Sub-escala P: somar os itens 3, 5, 7, 10, 14, 18. Sub-escala C: somar os itens 2, 4, 9, 11, 15, 16. Os escores variam entre 6 e 36 pontos; quanto maior o valor, maior a crença de que cada respectivo fator controle a sua saúde.

Cálculos preliminares para estimativa do tamanho da amostra foram dispensados, devido à ausência de consenso e fórmula matemática para determinar o tamanho amostral de um estudo piloto. Entretanto, a literatura reporta que 30 a 50 participantes em estudos piloto são suficientes [22].

Os dados foram duplamente digitados e validados no EpiData 3.1. Utilizou-se o programa IBM SPSS Statistics (versão 22.0) para realização da análise estatística. Foi realizada análise descritiva das variáveis observadas para obtenção de média, desvio padrão e frequências. A normalidade das variáveis quantitativas foi avaliada pelo teste de Shapiro-Wilk. A análise da correlação entre as variáveis foi verificada pelo teste de correlação de Spearman. Para a interpretação e inferência estatística utilizou-se um nível de confiança de $95 \%$ para um erro aleatório máximo até $5 \%(\mathrm{p} \leq 0,05)$.

\section{RESULTADOS}

A amostra foi composta por 37 idosos, sendo 22 mulheres e 15 homens, com idade mínima de 63 anos e máxima de 91 anos (média 73,41 77,25 ). A maior parte dos participantes possuía residência própria $(94,6 \%)$, saneamento básico $(94,6 \%)$ e tinha renda entre dois e cinco salários mínimos $(48,6 \%)$. As características sociodemográficas estão apresentadas na Tabela 1.

O total de dentes avaliados foi de 141. Desses, 86 estavam hígidos e o índice CPOD encontrado foi de $25,38 \pm 5,18$. O total de idosos que usavam prótese total removível foi de $26(70,3 \%)$. A média da pontuação total do OHIP-14 foi de 23,43 $\pm 10,25$. Os domínios que apresentaram maior e menor pontuação foram dor física $(4,78 \pm 2,01)$ e desvantagem social $(2,38 \pm 1,01)$ respectivamente (Tabela 2 ). Não houve perdas de questionários de qualidade de vida.

Em relação ao questionário para identificar problemas relacionados ao uso do álcool, sete $(18,9 \%)$ participantes não responderam ao questionário, 29 $(78,4 \%)$ foram classificados como abstêmios ou com baixo consumo de álcool e dois $(5,4 \%)$ como usuários de álcool. Todos responderam ao questionário ASSIST, sendo que 25 (67,6\%) foram classificados como uso ocasional de substâncias psicoativas e cinco $(13,5 \%)$ como dependentes (Tabela 3).

Do total de entrevistados, seis não responderam ao questionário de locus de controle de saúde. Dos 31 respondentes, $12(38,7 \%)$ foram classificados como locus externo, $11(35,5 \%)$ como locus acaso e oito $(25,8 \%)$ como locus interno (Tabela 4$)$.

As correlações estatisticamente significativas encontradas foram as seguintes: correlação positiva moderada entre locus interno e idade $\left(\mathrm{r}_{\mathrm{s}}: 0,428, \mathrm{p}=0,016\right)$, ou seja, quanto maior a idade dos participantes mais eles se julgavam responsáveis pela própria saúde; correlação positiva moderada entre limitação funcional e idade $\left(r_{s}: 0,397, p=0,015\right)$, indicando que o aumento da idade está associado a mais limitações funcionais; correlação positiva moderada entre locus externo e locus ao acaso $\left(\mathrm{r}_{\mathrm{s}}: 0,480, \mathrm{p}=0,006\right)$, ou seja, os idosos que tinham maior escore no locus externo de saúde também apresentavam alta pontuação no controle ao acaso de sua saúde; correlação negativa moderada entre limitação funcional e renda $\left(\mathrm{r}_{\mathrm{s}}:-0,332, \mathrm{p}=0,045\right)$, ou seja, quanto maior a limitação funcional menor a renda; correlação positiva moderada entre limitação funcional e locus interno $\left(\mathrm{r}_{\mathrm{s}}: 0,426, \mathrm{p}=0,017\right)$, isto é, quanto maior a limitação funcional, mais eles se julgavam responsáveis pela própria saúde; e correlação negativa moderada entre CPOD e incapacidade

Tabela 1. Características sociodemográficas dos 37 idosos participantes do estudo. Município de Diamantina, Minas Gerais, Brasil, 2015.

\begin{tabular}{|c|c|c|}
\hline Variável & Média $\pm D P$ & $\mathrm{n}(\%)$ \\
\hline Idade & $73,41 \pm 7,25$ & $37(100)$ \\
\hline $\begin{array}{l}\text { Estrutra familiar } \\
\text { Nuclear } \\
\text { Sem resposta }\end{array}$ & & $\begin{array}{l}13(35,1) \\
24(64,9)\end{array}$ \\
\hline Número de moradores por residência & $3,94 \pm 2,23$ & $37(100)$ \\
\hline $\begin{array}{l}\text { Tipo de residência } \\
\text { Própria } \\
\text { Aluguel }\end{array}$ & & $\begin{array}{c}35(94,6) \\
2(5,4)\end{array}$ \\
\hline $\begin{array}{l}\text { Saneamentobásico } \\
\text { Sim } \\
\text { Não }\end{array}$ & & $\begin{array}{c}35(94,6) \\
2(5,4)\end{array}$ \\
\hline $\begin{array}{l}\text { Escolaridade } \\
\text { Analfabeto } \\
\text { Ensino fundamental incompleto } \\
\text { Ensino fundam. Completo } \\
\text { Ensino médio incompleto } \\
\text { Ensino médio Completo } \\
\text { Ensino superior }\end{array}$ & & $\begin{aligned} 3 & (8,1) \\
5 & (13,5) \\
22 & (59,4) \\
2 & (5,4) \\
4 & (10,9) \\
1 & (2,7)\end{aligned}$ \\
\hline $\begin{array}{l}\text { Renda familiar } \\
\quad<1 \text { salário mínimo } \\
1 \text { a }<2 \text { salários } \\
2 \text { a }<5 \text { salários } \\
5 \text { a }<10 \text { salários }\end{array}$ & & $\begin{aligned} 2 & (5,4) \\
16 & (43,3) \\
18 & (48,6) \\
1 & (2,7)\end{aligned}$ \\
\hline
\end{tabular}

DP: desvio padrão. 
social ( $\left.\mathrm{r}_{\mathrm{s}}:-0,363, \mathrm{p}=0,032\right)$, em outras palavras, quanto maior o índice CPOD, menor a incapacidade social. Os demais resultados do teste de correlação estão explicitados na Tabela 5.

Tabela 2. Pontuação obtida por 37 idosos no OHIP-14 e subdomínios, no município de Diamantina, Minas Gerais, Brasil, 2015.

\begin{tabular}{l|c}
\hline \multicolumn{1}{c|}{ Variável } & Média \pm DP \\
\hline OHIP limitação funcional & $3,05 \pm 1,84$ \\
OHIP dor física & $4,78 \pm 2,01$ \\
OHIP desconforto psicológico & $3,84 \pm 2,41$ \\
OHIP incapacidade física & $3,16 \pm 2,00$ \\
OHIP incapacidade psicológica & $3,38 \pm 2,22$ \\
OHIP incapacidade social & $2,84 \pm 1,69$ \\
OHIP desvantagem social & $2,38 \pm 1,01$ \\
OHIP total & $23,43 \pm 10,25$ \\
\hline
\end{tabular}

DP: desvio padrão.

OHIP-14: Oral Health Impact of Profile (curto).
Tabela 3. Pontuação obtida por 37 idosos no AUDIT e ASSIST e respectivas classificações. Diamantina, Minas Gerais, Brasil, 2015.

\begin{tabular}{lcr}
\hline \multicolumn{1}{c}{ Variável } & Média \pm DP & $\mathbf{n}(\%)$ \\
\hline AUDIT $(\mathrm{n}=31)$ & & \\
Abstêmio/baixo consumo de álcool & $0,73 \pm 2,16$ & $29(93,5)$ \\
Usuário de álcool & & $2(6,5)$ \\
ASSIST ( $\mathrm{n}=37)$ & & \\
Uso ocasional & $2,58 \pm 4,36$ & $25(67,6)$ \\
Uso abusivo & & $7(18,9)$ \\
Dependência & & $5(13,5)$ \\
\hline
\end{tabular}

DP: desvio padrão

AUDIT: teste para identificação de problemas relacionados ao uso do álcool.

ASSIST: teste para triagem do envolvimento com álcool, cigarro e outras substâncias.

Tabela 4. Pontuação obtida pelos idosos no locus de saúde. Diamantina, Minas Gerais, Brasil, $2015(n=31)$.

\begin{tabular}{l|r|r}
\hline \multicolumn{1}{|c|}{ Variável } & Média $\pm \mathbf{D P}$ & n (\%) \\
\hline Locus interno & $13,29 \pm 5,05$ & $8(25,8)$ \\
\hline Locus externo & $16,26 \pm 7,67$ & $12(38,7)$ \\
Locus acaso & $16,23 \pm 6,26$ & $11(35,5)$ \\
\hline
\end{tabular}

DP: desvio padrão.

Tabela 5. Correlação entre índice CPOD e variáveis psicossociais na população idosa de Diamantina, Minas Gerais, Brasil, $2015(n=37) \ldots$

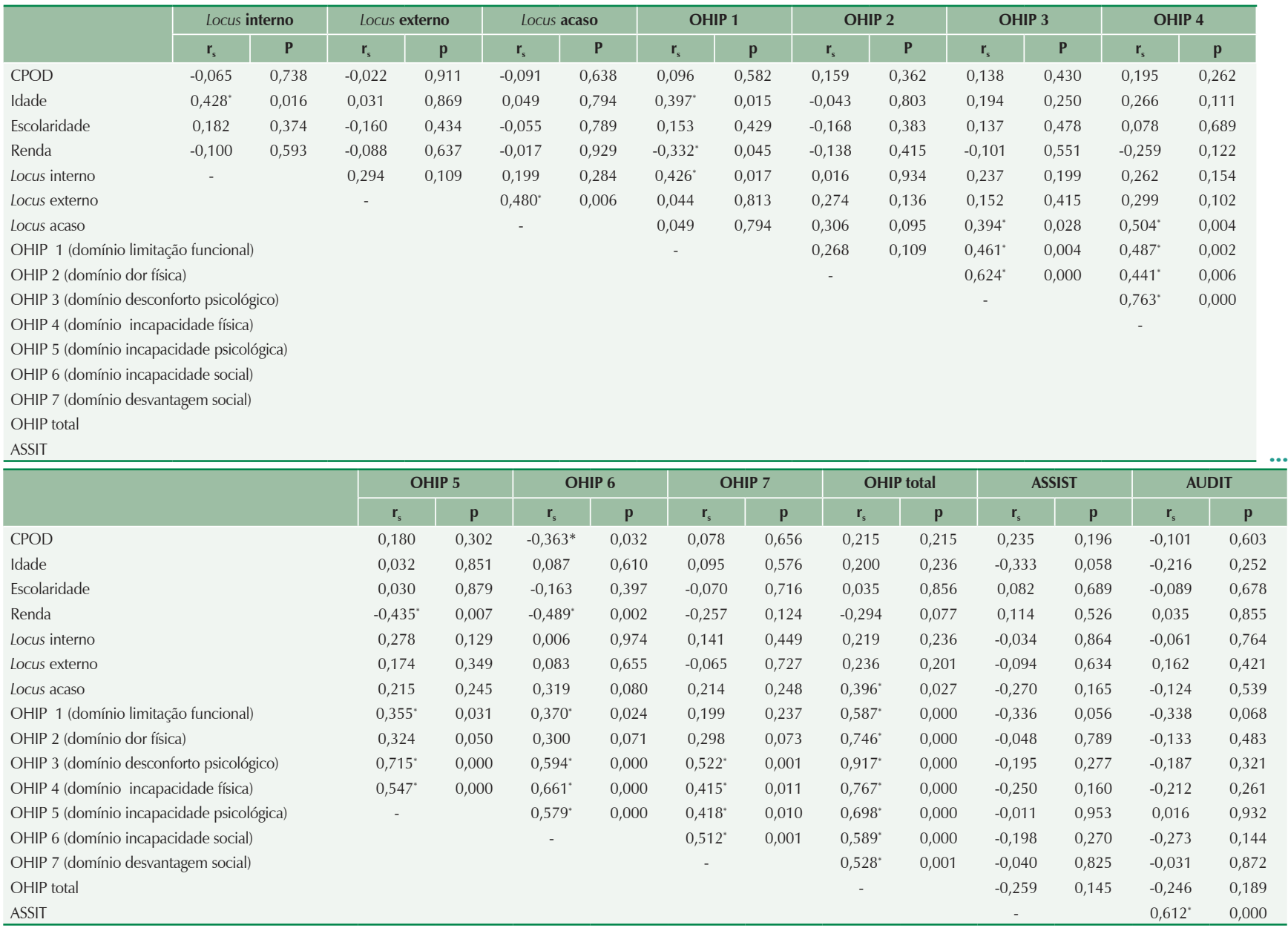

$r_{s}$ : coeficiente de correlação de Spearman. ASSIST: teste para triagem do envolvimento com álcool, cigarro e outras substâncias. AUDIT: teste para identificação de problemas relacionados ao uso do álcool. CPOD: índice de dentes cariados, perdidos e obturados.

* Estatisticamente significante. 


\section{DISCUSSÃO}

O presente estudo piloto demonstrou que a saúde bucal (representada pelo índice CPOD) e alguns fatores psicossociais estão moderadamente correlacionados entre si, e são capazes de interferir diretamente na saúde do idoso. Essa população percebe a importância da saúde bucal em suas vidas, principalmente nas formas dos domínios físico, social e psicológico. Quando esses fatores não atendem às expectativas do indivíduo idoso, podem ser desencadeadas sensações de insegurança, ansiedade e redução da autoestima. Dessa forma, a qualidade de vida está intimamente relacionada ao grau de satisfação pessoal momentânea, sendo mutável e totalmente influenciada pelo estado socioeconômico, cultural, físico e psicológico.

A sociedade brasileira encontra-se em constante transformação (econômica, política e social) e expõe o indivíduo diariamente a fatores que podem influenciar diretamente a saúde e a qualidade de vida. Deve-se investir na promoção da saúde junto às populações para reduzir os efeitos de determinados fatores responsáveis por sua morbidade e mortalidade, assim como para evitar doença e promover saúde [23].

No presente estudo piloto, a maioria dos idosos possuía residência própria, saneamento básico e renda entre dois e cinco salários mínimos. Um estudo feito por Joia et al. [24] reportou que apenas $18,1 \%$ dos idosos tinham renda superior a dois salários mínimos. Esse contraste pode ter ocorrido devido ao fato de que apenas 9,3\% haviam concluído o ensino superior naquele estudo.

Em relação às condições de saúde bucal, o alto índice CPOD e a alta taxa de edentulismo encontrada neste estudo corroboram achados de vários outros estudos anteriores brasileiros [25-27]. Estes dados confirmam o modelo de saúde brasileiro, baseado historicamente em ações terapêuticas, muitas vezes envolvendo extrações dentárias [27]. E assim, percebese a necessidade de mudança para uma política de saúde pública que seja voltada para a prevenção e promoção de saúde bucal, priorizando a saúde no lugar da doença.

A baixa renda refletiu em impacto negativo na qualidade de vida dos idosos do presente estudo, o que está em acordo com a literatura, em que idosos com menor renda tinham qualidade de vida comprometida [27-29]. A presença de dor física também influenciou negativamente a qualidade de vida dos idosos neste estudo, assim como em estudo anterior [27], reforçando a importância de que a prática odontológica seja preventiva, evitando a dor causada por problemas dentários. Durante muito tempo, o Brasil teve como alvo de promoção e prevenção de saúde bucal crianças pré-escolares, excluindo desse tipo de cuidados os adultos e idosos, que acabam por receber, em sua maior parte, somente atendimento de urgências odontológicas [3].

A maioria dos idosos declarou-se como abstêmio ou com baixo consumo do álcool e usuários ocasionais de substâncias psicoativas, o que parece ser um fator de proteção à saúde, levando em conta que uma grande parte dos idosos fazem uso regular de medicamentos que possuem interação com álcool e substâncias psicoativas [30]. Este achado parece respaldar o fato dos idosos serem menos vulneráveis a acidentes e brigas [31], o que contribui para a qualidade de vida. Os baixos consumos de álcool e outras substâncias entre idosos parecem estar de acordo com vários outros estudos realizados anteriormente [32-34].

Um constructo amplamente usado para discussões sobre variáveis relacionadas à qualidade de vida é o locus de controle da saúde [35]. Discutir qualidade de vida é uma questão multidimensional, na medida em que depende de diversas variáveis do contexto do indivíduo. Locus de controle é um dos fatores relacionados ao bem-estar subjetivo, o qual é um dos componentes do conceito de qualidade de vida [36]. Indivíduos com locus de controle interno da saúde acreditam que sua saúde é influenciada por seus próprios esforços e habilidades, e em função disso, são mais propensos à adesão de atividades de promoção de saúde. Pessoas com um locus de controle de saúde externo são menos susceptíveis às mensagens de promoção da saúde, mas podem aderir com mais probabilidade a intervenções que envolvam a ação de profissionais de saúde $[37,38]$. No caso de idosos, os que apresentam como predominante o locus de controle interno possuem um estilo de vida mais ativo e uma percepção maior da qualidade de vida [36]. A minoria dos idosos entrevistados neste estudo relatou o locus de controle de saúde interno, mas uma proporção relevante relatou o locus de saúde externo. Este resultado sinaliza para a importância da atuação de profissionais de saúde em promover, proteger e recuperar a autonomia dos idosos quanto aos cuidados com saúde desta população.

Freitas e Py [13] argumentam que a autonomia e a independência, especialmente entre os idosos, são bons indicadores de saúde e consequentemente de qualidade de vida. Os autores defendem que a incapacidade real ou percebida de operar sobre o meio físico e social produz nas pessoas uma sensação de fracasso. Nesse sentido, as pessoas que atribuem seu fracasso a deficiências pessoais, generalizadas e duradouras, podem ser com maior probabilidade acometidas de 
sentimentos de impotência, desamparo e desesperança. Sendo assim, quanto maior a percepção de controle pessoal e autonomia para tomadas de decisão, mais intensos são os sentimentos de satisfação e maior é a probabilidade de um estilo de vida ativo (por exemplo, nos cuidados com a saúde); consequentemente, maior deve ser a percepção de qualidade de vida.

O presente estudo encontrou uma correlação moderada e positiva entre locus interno e idade. Esse resultado corrobora com pesquisas que documentam benefícios à saúde e à qualidade de vida da presença do locus controle da saúde interno, pois favorece a emissão de comportamentos positivos para a saúde que se tornam particularmente importantes no decorrer da longevidade, como a gestão de doenças crônicas e a adesão a um estilo de vida saudável $[36,39,40]$.

Foram identificadas também uma correlação moderada e positiva entre o domínio de qualidade de vida chamado de limitação funcional e a idade e uma correlação moderada e negativa entre índice CPOD e o domínio de qualidade de vida chamado de incapacidade social. Estes resultados podem refletir a importância do desenvolvimento psicológico e social durante o envelhecimento, concomitantemente ao aumento da necessidade de tratamento restaurador e protético [10]. À medida em que a pessoa envelhece, há uma tendência a aumentar a necessidade de tratamento odontológico, relacionada a perdas das funções do sistema estomatognático, como mastigar, deglutir e conversar, além de aspectos de estética e socialização.
Também foi encontrada uma correlação moderada e negativa entre a o domínio de qualidade de vida limitação funcional e renda familiar, o que pode sugerir que pessoas idosas em circunstâncias economicamente desfavoráveis têm menos autonomia para pagar por tratamento odontológico, e podem com maior probabilidade ter comprometimentos com as funções bucais e consequentes prejuízos funcionais.

Os resultados reportados aqui apresentam relevantes informações para o desenvolvimento de programas de promoção de saúde, em especial ações em saúde bucal, em grupos de idosos. O presente estudo piloto tem algumas limitações, como a impossibilidade de emprego de análises de regressões devido ao pequeno número de participantes. Em adição, este estudo caracteriza-se por ser transversal, impossibilitando o estabelecimento de relação de causalidade entre as variáveis estudadas. São necessárias pesquisas longitudinais, com tamanho amostral maior e análises estatísticas robustas, para determinar melhor a direção e força das correlações.

Dentre as dimensões investigadas, concluiu-se que qualidade de vida, locus de controle de saúde e índice CPOD foram fatores correlacionados nesta população de idosos. É importante ressaltar as prováveis influências positivas que estas características têm sobre a manutenção da qualidade de vida da pessoa idosa. Com isso, enfatiza-se a importância da atuação de profissionais de saúde e, mais especificamente, de saúde bucal, na promoção de um envelhecimento saudável.

\section{REFERÊNCIAS}

1. Mendes DC, Poswar FO, Oliveira MV Haikal DS, da Silveira MF, MartinsAM, De Paula AM. Analysis of sociodemographic and systemic health factors and the normative conditions of oral health care in a population of the Brazilian elderly. Gerodontology. 2012;29(2):206-14. http://dx.doi.org/10.1111/j.1741-2358.2010.00446.x

2. Chaimowicz F. A saúde dos idosos brasileiros às vésperas do século XXI: problemas, projeções e alternativas. Rev Saude Publica. 1997;31(2):184-200. http://dx.doi.org/10.1590/S0034-89101997000200014

3. Moreira RS, Nico LS, Sousa ML. Factors associated with subjective need for dental treatment in elderly Brazilians. Cad Saude Publica. 2009;25(12):2661-71. http://dx.doi.org/10.1590/S0102-311X2009001200013

4. Minayo MCS, Hartz ZMA, Buss PM. Qualidade de vida e saúde: um debate necessário. Cienc Saude Colet 2000;5(1):7-18. http://dx.doi. org/10.1590/S1413-81232000000100002

5. Fleck MPA. A Avaliação de qualidade de vida: guia para profissionais da saúde. Porto Alegre: Artmed; 2008.

6. Seidl EM, Zannon CM. Qualidade de vida e saúde: aspectos conceituais e metodológicos. Cad Saude Publica. 2004;20(2):580-8. http:// dx.doi.org/10.1590/S0102-311X2004000200027

7. Lima-Costa MF, Veras R. Saúde pública e envelhecimento. Cad Saúde Pública. 2003;19(3):700-1. http://dx.doi.org/10.1590/S0102311X2003000300001

8. Atkinson RM. Alcohol problems of the eldery. Alcohol Alcohol. 1987;22(4):415-7.

9. Greene CA, Murdock KK. Multidimensional control beliefs, socioeconomic status, and health. Am J Healt Behav. 2013;37(2):227-37. http://dx.doi.org/10.5993/AJHB.37.2.10

10. Peker K, Bermek G. Oral health: locus of control, health behavior, self-rated oral health and socio-demographic factors in Istanbul adults. Acta Odontol Scand. 2011;69(1):54-64. http://dx.doi.org/10.3109/00016357.2010.535560 
11. Coleta MF. Atribuição de causalidade: teoria e pesquisa. Rio de Janeiro: Fundação Getúlio Vargas; 1982.

12. Furnham A, Steele H. Measuring locus of control: a critique of general, children's, health and workrelated locus of control questionnaires. Br J Psychol. 1993;84(4):443-79. http://dx.doi.org/10.1111/j.2044-8295.1993.tb02495.x

13. Freitas EV, Py L (Eds.). Tratado de geriatria e gerontologia. 3를. Rio de Janeiro: Guanabara Koogan; 2011.

14. Cypriano S, Pecharki GD, Souza MLR, Wada RS. A saúde bucal de escolares residentes em locais com ou sem fluoretação nas águas de abastecimento público na região de Sorocaba, São Paulo, Brasil. Cad Saude Publica. 2003;19(4):1063-71. http://dx.doi.org/10.1590/ S0102-311X2003000400028

15. Organização Mundial De Saúde. Levantamentos básicos em saúde bucal, 4aㅗ ed. São Paulo: Santos; 1999.

16. Oliveira BH, Nadanovsky P. Psychometric properties of the Brazilian version of the oral health impact profile-short form. Community Dent Oral Epidemiol. 2005;33(4):307-14. http://dx.doi.org/10.1111/j.1600-0528.2005.00225.x

17. Lima CT, Freire AC, Silva AP, Teixeira RM, Farrell M, Prince M. Concurrent and construct validity of the AUDIT in urban Brazilian sample. Alcohol Alcohol. 2005;40(6):584-9. http://dx.doi.org/10.1093/alcalc/agh202

18. Babor TF, Higgins-Biddle JC, Saunders JB, Monteiro MG, World Health Organization. Department of Mental Health and Substance Dependence. AUDIT: the Alcohol Use Disorders Identification Test: guidelines for use in primary health care. 2nd. Geneva: World Health Organization; 2001.

19. Henrique IF, Micheli D, Lacerda RB, Lacerda LA, Formigoni ML. Validation of the Brazilian version of alcohol, smoking and substance involvement scrrening test (ASSIST). Rev Assoc Med Bras. 2004;50(2):199-206. http://dx.doi.org/10.1590/S0104-42302004000200039

20. Brandão IM, Arcieri RM, Sundfeld ML, Moimaz SA. Cárie precoce: influência de variáveis sociocomportamentais e do locus de controle da saúde em um grupo de crianças de Araraquara, São Paulo. Cad Saude Publ. 2006;22(6):1247-56. http://dx.doi.org/10.1590/S0102311X2006000600014

21. Acharya S, Pentapati KC, Singh S. Influence of socioeconomic status on the relationship between locus of control and oral health. Oral Health Prev Dent. 2011;9(1):9-16.

22. Melody AH. Considerations in Determining Sample Size for Pilot Studies. Res Nurs Health 2008;31(2):180-91. http://dx.doi.org/10.1002/ nur.20247

23. Figueiredo JP, Cardoso SM. Perceived health in the Portuguese population aged $\geq 35$. Rev Saude Publica. 2014;48(3):406-27. http:// dx.doi.org/10.1590/S0034-8910.2014048005242

24. Joia LC, Ruiz T,Donalisio MR. Condições associadas ao grau de satisfação com a vida entre a população de idosos. Rev Saude Publica 2007;41(1):131-8. http://dx.doi.org/10.1590/S0034-89102007000100018

25. Moreira RS, Nico LS, Tomita NE, Ruiz T. Oral health of Brazilian elderly: a systematic review of epidemiologic status and dental care access. Cad Saude Publica 2005;21:1665-75. http://dx.doi.org/10.1590/S0102-311X2005000600013

26. Hugo FN, Hilgert JB, Sousa ML, Silva DD, Pucca Jr GA. Correlates of partial tooth lossand edentulism in the Brazilian elderly. Comm Dent Oral Epidemiol. 2007;35(3):224-32. http://dx.doi.org/10.1111/j.0301-5661.2007.00346.x

27. Ulinski KG, do Nascimento MA, Lima AM, Benetti AR, Poli-Frederico RC, Fernandes KB, Fracasso ML, Maciel SM. Factors related to oral health-related quality of life of independent brazilian elderly. Int J Dent. 2013;2013:705047. http://dx.doi.org/10.1155/2013/705047

28. Slade GD. Derivation and validation of a short-form oral health impact profile. Comm Dent Oral Epidemiol. 1997;25(4):284-90. http:// dx.doi.org/10.1111/j.1600-0528.1997.tb00941.x

29. Sanders AE, Slade GD, Lim S, Reisine ST. Impact of oral disease on quality of life in the US and Australian populations. Comm Dent Oral Epidemiol. 2009;37(2):171-181. http://dx.doi.org/10.1111/j.1600-0528.2008.00457.x

30. Paskulin LMG, Vianna LAC. Perfil sócio-demográfico e condições de saúde auto-referidas de idosos de Porto Alegre. Rev Saúde Pública.2007;41(5):757-68. http://dx.doi.org/10.1590/S0034-89102007000500010

31. Lima MC, Simão MO, Oliveira JB, Cavariani JB, Tucci AM, Kerr-Correa F. Alcohol use andfallsamongtheelderly in Metropolitan São Paulo, Brazil. Cad Saúde Públ. 2009;25(12):2603-11. http://dx.doi.org/10.1590/S0102-311X2009001200007

32. Mendoza-Sassi RA, Beria JU. Prevalence of alcohol use disorders and associated factors: a population-based study using AUDIT in Southern Brazil. Addiction 2003;98(6):799-804. http://dx.doi.org/10.1046/j.1360-0443.2003.00411.x

33. Almeida-Filho N, Lessa I, Magalhães L, Araújo MJ, Aquino E, Kawachi I, et al. Alcohol drinking patterns by gender, ethnicity, and social class in Bahia, Brazil. Rev Saude Publica. 2004;38(1):45-54. http://dx.doi.org/10.1590/S0034-89102004000100007

34. Laranjeira R, Pinsky I, Zaleski M, Caetano R. II levantamento nacional sobre os padrões de consumo de álcool na população brasileira. Brasília: Secretaria Nacional Antidrogas; 2007.

35. Wallston BS, Wallston KA, Kaplan GD, Maides SA. Development and validation of the health locus of control (HLC) scale. J Consul Clin Psychol; 1976;44(4):580-5. http://dx.doi.org/10.1037/0022-006X.44.4.580

36. Timm LA, Argimon IIL, Wendt GW. Correlation between domains of quality of life and locus of health control in community resident elderly. Scientia Medica 2011;21(1):9-13.

37. Galloway RD. Health promotion: causes, beliefs and measurements. Clin Med Res. 2003;1(3):249-58. http://dx.doi.org/10.3121/cmr.1.3.249

38. Haber D. Health promotion and aging. Practical applications for health professionals, 4th ed. New York: Springer Publishing Company; 2007.

39. Markey C, Markey P, Schneider C, Brownlee S. Marital status and health beliefs: Different relations for men and women. Sex Roles. 2005;53(5):443-51. http://dx.doi.org/10.1007/s11199-005-6767-y

40. Robison-Whelen S, Bodenheimer C. Health practices of veterans with unilateral lower-limb loss: Identifying correlates. J Rehabil Res Dev. 2004;41(3):453-60. http://dx.doi.org/10.1682/JRRD.2004.03.0453 